\title{
Trivium
}

Revue franco-allemande de sciences humaines et sociales - Deutsch-französische Zeitschrift für Geistesund Sozialwissenschaften

$30 \mid 2019$

La constitution au tournant des XXe et XXle siècles

\section{La doctrine de droit public allemande et les débats européanisés de la science du droit}

Caractéristiques, compétences centrales et obstacles à sa réception

\section{Matthias Jestaedt}

Traducteur : Olivier Joop

\section{OpenEdition \\ Journals}

Édition électronique

URL : http://journals.openedition.org/trivium/6896

DOI : $10.4000 /$ trivium. 6896

ISSN : 1963-1820

Éditeur

Les éditions de la Maison des sciences de l'Homme

\section{Référence électronique}

Matthias Jestaedt, «La doctrine de droit public allemande et les débats européanisés de la science du droit », Trivium [En ligne], 30 | 2019, mis en ligne le 18 décembre 2019, consulté le 08 septembre 2020. URL : http://journals.openedition.org/trivium/6896 ; DOI : https://doi.org/10.4000/trivium.6896

Ce document a été généré automatiquement le 8 septembre 2020.

\section{(c) (i) (9)}

Les contenus des la revue Trivium sont mis à disposition selon les termes de la Licence Creative Commons Attribution - Pas d'Utilisation Commerciale - Pas de Modification 4.0 International. 


\title{
La doctrine de droit public allemande et les débats
}

\section{européanisés de la science du droit}

Caractéristiques, compétences centrales et obstacles à sa réception

\author{
Matthias Jestaedt
}

Traduction : Olivier Joop

\section{NOTE DE L'ÉDITEUR}

Nous remercions M. Matthias Jestaedt et la maison d'édition Mohr Siebeck de nous avoir accordé l'autorisation de traduire ce texte pour le présent numéro.

Wir danken Herrn Matthias Jestaedt und dem Verlag Mohr Siebeck für die freundliche Genehmigung, diesen Artikel in französischer Übersetzung zu publizieren.

\section{Une perspective inter-doctrinale}

1 Lorsqu'on s'interroge sur les défis actuels auxquels la science du droit public en Allemagne doit faire face, on mentionne surtout - certes pas uniquement - les défis dont les origines sont extérieures aux frontières de l'Allemagne. Les mots-clés les plus souvent cités sont l'européanisation et la mondialisation, qui constituent les raisons et le moteur d'une remise en cause générale de l'état et de l'évolution de la discipline, en dépit du fait qu'elle a réussi à prendre en compte certains besoins d'adaptation et de réorientation aussi bien quotidiens que ciblés et spécifiques. La supranationalisation croissante de la puissance publique, le manque persistant d'une science du droit de l'Union " autochtone ", c'est-à-dire étudiant le droit de l'Union dans une perspective commune et globale, et non dans une perspective sectorielle et nationale - donc de fait selon 27 perspectives plus ou moins sans liens les unes avec les autres -, le fait que dans le cadre du processus de formation de l'Union, un rôle significatif revient 
naturellement aux sciences juridiques des États membres, mais également la montée en puissance du système de protection des droits par les juges européens de Strasbourg : il y a là un ensemble de facteurs qui, parmi d'autres, poussent les sciences juridiques nationales du droit public à un (re)positionnement stratégique. Dès lors, il n'est guère étonnant que ces dernières années, on tente de plus en plus de déterminer la place des sciences juridiques allemandes, en mettant l'accent sur des détails différents. On s'interroge ainsi, pour ne citer que trois exemples phares, sur la place de la science constitutionnelle allemande dans le contexte des changements que connaît la communauté scientifique internationale ${ }^{1}$, on essaie de cerner le rôle de la science juridique allemande au sein de l'espace juridique européen ${ }^{2}$ et on développe des réflexions - avec comme idée directrice le «juriste européen» - portant sur la formation des juristes et sur la culture juridique en Allemagne ${ }^{3}$.

2 Les présentes réflexions s'inscrivent dans ces efforts, mais elles adoptent cependant une perspective dans l'ensemble plus modeste et plus spécifique. Selon la perspective inter-doctrinale adoptée ici, l'attention se porte en priorité sur la science du droit public développée en Allemagne par rapport à et en comparaison avec ses homologues dans d'autres pays (en particulier les États membres de l'Union européenne). En revanche, le point de vue interdisciplinaire, lequel s'intéresse principalement à la question de la réception et de la compatibilité de la doctrine juridique avec des disciplines autres que juridiques, ne sera traité que marginalement. De même, la question intra-disciplinaire qui consiste à s'interroger sur la place et l'évolution de la science du droit public par rapport à celles des sciences du droit privé et du droit pénal ne sera pas approfondie $\mathrm{ici}^{4}$. Une troisième remarque restrictive : lorsque, dans les développements qui suivent, il sera question de la science du droit public (ou encore de la théorie du droit public), le terme renverra au premier chef à la recherche, et il ne sera fait référence à l'enseignement que là où ce dernier revêt une importance structurante pour la première. En quatrième lieu, le fait que, ce faisant, une telle démarche soit réductrice et implique une approche qui se concentre sur les "grandes lignes » et dépasse l'analyse détaillée de telle ou telle argumentation particulière, de tel ou tel modèle concret ou encore de tel ou tel institut de recherche en particulier est dû d'une part au nombre limité de pages disponibles pour la présente contribution et d'autre part à un souci de clarté.

3 En dernier lieu, contrairement à la démarche suivie par la majorité des autres contributions sur ce sujet, il ne s'agira pas tant pour la présente contribution de formuler des remèdes destinés à combler des carences, d'avancer des recommandations pour des actions futures, ni même de thématiser les projets de révisions ou d'adaptation de la science du droit public en Allemagne. Non que je cherche à affirmer ici qu'il faille s'opposer aux revendications et aux propositions avancées. Ici, l'idée directrice est qu'avant toute ambition concrète de changement et d'adaptation destinée à remédier aux impasses ou aux raccourcis, à des approches méthodologiques désuètes ou des orientations de la discipline inadaptées à l'époque contemporaine, voire à surmonter le "nationalisme méthodologique ${ }^{5}$ ", il faut dresser - encore et toujours un état des lieux (plus) précis de ce qui caractérise spécifiquement l'apport de la science du droit public allemande face aux défis internationaux et, surtout, européens. Deux questions seront au centre de la présente analyse. Quelles sont les caractéristiques principales et quelle est l'identité (Markenkern) de la science du droit public en Allemagne ? Dans quelle mesure, ensuite, ces caractéristiques et cette identité 
facilitent-elles ou rendent-elles au contraire plus difficiles les échanges et le rapprochement avec les sciences du droit public dans d'autres pays ? Les réponses à ces deux questions fourniront, tantôt directement, tantôt indirectement, des indices pour répondre aux questions qui en découlent - et qui ne seront pas traitées ici -, visant à déterminer, d'une part, les orientations fondamentales et les compétences centrales que la science du droit public allemande devra posséder pour être en mesure de marquer de son empreinte la science du droit public commune européenne naissante, ainsi que, d'autre part, le rôle et l'importance de la première au sein de la seconde.

\section{La dépendance au sentier dogmatique : la science du droit comme dogmatique}

\section{Penser en dogmaticien}

Quiconque considère la structure et à la texture fondamentales de la science du droit public allemand au sein des autres sciences (européennes) du droit public sera inévitablement confronté à l'empreinte fondamentale centrale de cette science - dans ce contexte, il est également possible de parler d'une dépendance au sentier -, bien qu'il ne s'agisse pas là d'une spécificité de la littérature juridique en Allemagne, mais plus largement une caractéristique de la science et de la culture juridiques allemandes dans leur ensemble. En Allemagne, la dogmatique règne par une " présence écrasante ${ }^{6}$ ». La dogmatique comme mode de pensée et comme structure d'une discipline scientifique est certes d'origine indubitablement civiliste, mais l'approche dogmatique marque de son empreinte la doctrine juridique dans son ensemble et ce, depuis plus d'un siècle ${ }^{7}$. La division tripartite du monde juridique en Allemagne - que ce soit en ce qui concerne les débats scientifiques, l'enseignement à l'université ou encore la subdivision au sein des unités d'enseignement et de recherche en instituts et chaires -, fondée sur les trois " piliers » du droit public, du droit privé et du droit pénal, existe grâce à la dogmatique, qui entend ainsi reproduire la tripartition du droit en vigueur en Allemagne. Sans spécialisation dans une matière dogmatique (et sans avoir rédigé au moins soit la thèse de doctorat, soit la thèse pour l'obtention de l'habilitation universitaire dans un domaine touchant à la dogmatique), il est difficile d'envisager de réussir en tant qu'enseignant-chercheur en Allemagne (et dans les pays germanophones). La discipline guidant la pratique scientifique en matière juridique accède au rang de discipline principale, transformant ainsi les "sciences juridiques", au pluriel, en « science juridique », au singulier.

Certains auteurs vont alors jusqu'à assimiler - consciemment ou non - science juridique et dogmatique juridique ${ }^{8}$. Probablement, aucun autre aspect que celui de la dépendance au sentier de la dogmatique juridique n'est mieux à même d'illustrer les splendeurs et les misères de la science juridique allemande sous l'angle de sa réception au-delà des frontières nationales.

\section{La dogmatique comme forme de communication commune entre science du droit et pratique du droit}

6 Le rôle prédominant incontesté de la dogmatique au sein des disciplines juridiques s'explique tout d'abord par le fait que, forte de l'orientation vers la pratique - ou mieux 
encore, de l'inclusion de la pratique - qui lui est consubstantielle, la dogmatique constitue tant le produit que le catalyseur d'un système mêlant théorie et pratique, un système propre à la doctrine juridique germanophone et produisant ses effets tant sur la recherche que sur l'enseignement. Depuis toujours, la dogmatique a su marier de manière presque symbiotique la pratique et la science du droit. Elle offre la base à partir de laquelle la pratique et la science du droit communiquent intensément et s'enrichissent mutuellement. Ainsi, la forme de publication juridique consubstantielle à la dogmatique est celle du code ou de la loi commentés et donnant des indications pour l'application du droit, une forme de publication qui, dans le contexte international, peut être considérée comme une particularité allemande (ou germanophone) dans le discours de la science juridique - ou, plus prudemment peut-être, comme une contribution spécifiquement allemande (ou germanophone) à un discours de la science du droit de plus en plus internationaliste. La plupart des revues juridiques témoignent elles aussi de la relation symbiotique entre science et pratique véhiculée par la dogmatique 9 . Dès lors qu'elles n'ont pas valeur d'archives ou qu'elles ne sont exclusivement consacrées à des recherches sans rapport avec la dogmatique, les publications de ces revues comportent typiquement un article, qui contient la contribution scientifique à proprement parler, et une partie consacrée à la jurisprudence, dans laquelle sont citées textuellement les décisions récentes les plus importantes. Dans les articles scientifiques de type dogmatique, ainsi que dans les arrêts des juridictions (suprêmes), les références (et citations) à des décisions juridictionnelles et à des contributions de la doctrine se retrouvent indistinctement côte à côte, souvent comme éléments destinés à étayer un même raisonnement. Ce qui est communément appelé la "doctrine dominante» - qui tient compte, outre des contributions doctrinales, également de la pratique juridique - ainsi que la «jurisprudence constante» d'une juridiction supérieure, voire suprême, constituent très souvent un argument de premier choix pour soutenir un raisonnement dans les débats scientifiques.

7 Ce qui, vu de l'extérieur, peut apparaître comme une transgression des frontières n'est pas perçu comme tel par les acteurs concernés, qui, de part et d'autre de cette frontière parlent le même langage, celui de la dogmatique, à laquelle ils ont été formés dans le cadre de leurs études et qu'ils emploient depuis lors, même si cet emploi peut servir à des fins certes pas tout à fait identiques ${ }^{10}$. Cela est particulièrement manifeste pour quiconque s'intéresse à l'objectif que doit atteindre tout étudiant qui reçoit une formation juridique, sanctionnée par deux examens (d'État) en droit : cet objectif qui est formulé en termes on ne peut plus clairs au $\$ 5$ de loi allemande relative au statut des magistrats, n'est autre que remplir «les conditions d'accès à la magistrature du siège ». Le titulaire des deux examens d'État en droit, celui qui est " habilité à accéder à la magistrature du siège », est désigné par le terme de « juriste pleinement qualifié » (« Volljurist »).

\section{Une production systématique du droit : le mode opératoire de la dogmatique}

8 En substance, la dogmatique juridique consiste à travailler (aufbereiten) - dans une approche systématique s'appuyant sur les formes et les outils de la science - le droit positif en vue de son application. À cette fin, la dogmatique s'est dotée d'un mode opératoire grâce auquel la science du droit et le droit lui-même se trouvent liés d'une 
manière à la fois particulière et féconde. Ainsi, l'orientation scientifique de la pratique juridique et l'orientation pratique de la science juridique vont de pair.

9 La manière de procéder se compose de trois opérations distinctes, du moins sur le plan de l'analyse : d'abord une décontextualisation par abstraction, ensuite l'établissement d'un système cohérent, puis une concrétisation au moyen d'une application concrète par déduction logique :

10 (1) La première étape consiste à traiter le cas juridique étudié d'une manière à pouvoir l'utiliser à des fins de dogmatique, c'est-à-dire de formuler des principes et des concepts dogmatiques. En bref, cette phase consiste à décontextualiser le phénomène juridique choisi, c'est-à-dire d'une part en lui ôtant au cas et aux faits concrets qu'il concerne les références qu'il contient, et d'autre part en l'extrayant par principe de l'environnement normatif concret de droit positif auquel il se rattache. Si le phénomène juridique en question est ainsi « apuré » de sa consistance, il ne reste plus que l'extrait ou l'essence de la signification de la norme. La décontextualisation sert, tout comme l'étape suivante, à la standardisation, la rationalisation et la stabilisation d'attentes normatives. De cette manière, les catégories dogmatiques dégagées assument une fonction de mémorisation et d'orientation lors de l'établissement d'une construction juridique.

11 (2) Pour que ces catégories puissent assurer cette fonction, elles doivent toutefois encore être soumises au processus de systématisation à proprement parler, par une démarche donnant une cohérence à leur contenu. Les règles de droit, largement dépourvues de leurs éléments contingents, sont placées en relations - le plus souvent hiérarchisées - si possible sans contradictions et dans leur intégralité. Ensemble, elles forment le système dogmatique qui, à son tour, leur confère un rang et une force. Dans ce système - entendu, nota bene, dans un sens matériel - se concrétise (le plus souvent implicitement) l'idée d'ordre juridique. L'ensemble de règles de la dogmatique absorbe le droit positif à un niveau d'abstraction plus élevé ${ }^{11}$. Le droit dans son ensemble, de même que ses normes codifiées (par exemple, le code civil, le code pénal ou la Loi fondamentale) sont perçus, d'une part, comme une «structure homogène possédant un sens propre » et, d'autre part, comme un " réseau de normes à concrétiser ${ }^{12}$ ". La notion de système - au sens matériel - qui, ce faisant, sert de point de référence est, comparée aux autres possibilités, la plus ambitieuse. Tout d'abord, elle va bien au-delà de la simple " systématicité ", qui, dans l'épistémologie moderne, est considérée comme une marque de scientificité et constitue un indicateur de quantité et qualité d'un ensemble relationnel dont la validité n'a pas (encore) été infirmée ${ }^{13}$. Cependant, la notion de système entendue dans un sens visant uniquement l'aspect de la procédure et des compétences et qui entend par le terme d'unité (du système) l'absence de contradictions au sein du contexte destiné à déterminer la compétence pour la création de normes de droit et dans lequel des règles de droit peuvent être adoptées ou abrogées, demeure en-deçà de la notion de système au sens matériel, qui vise à établir un ordre normatif exempt de contradictions au regard de son contenu et de sa valeur.

(3) Le système ainsi créé déploie son plein effet - à travers chacun de ses énoncés dans son processus d'application à la signification concrète et contingente de la norme (et du cas concret). Dans le cadre d'un processus de (re)concrétisation des conclusions dogmatiques dégagées, la norme de droit positif déterminante pour trancher un litige concret fait l'objet d'une interprétation dont la marge est délimitée par le système. Le contenu et la place de la règle de droit à appliquer concrètement peuvent être 
déterminés au moyen d'un raisonnement déductif et systématique. La science se retransforme alors en droit. Le circuit de rationalisation ainsi bouclé garantit la scientifisation de la création de normes de droit - et en même temps le caractère indispensable, auto-stabilisateur de la dogmatique juridique.

\section{L'imbrication de la science du droit constitutionnel et du droit administratif au sein de la science du droit public (Staatsrechtslehre)}

13 Lorsqu'il est question en Allemagne de la science du droit public, est visé - tant par la discipline scientifique que par les scientifiques eux-mêmes - le droit régissant l'État. La référence à l'État dans le terme allemand pour le droit public, le «Staatsrecht », n'est de nos jours majoritairement plus perçue comme une auto-description programmatique et militante. Elle est utilisée comme la désignation traditionnelle de la discipline qui regroupe la science du droit constitutionnel et la science du droit administratif et qui inclut de plus en plus la science du droit européen (dans la mesure où ce dernier comporte des éléments de droit public, c'est-à-dire de droit constitutionnel et de droit administratiff ${ }^{14}$. La manifestation la plus visible de cette communauté disciplinaire et personnelle est l'Association des professeurs allemands de droit public, fondée en 1922, qui regroupe en son sein la quasi-totalité des universitaires allemands, autrichiens et suisses(-allemands) qui sont titulaires d'une habilitation dans l'une des deux disciplines ${ }^{15}$.

14 Bien que, sous l'angle de la distinction entre droit public et droit privé, la doctrine constitutionnelle (tout comme celle du droit européen) ne puisse, en y regardant de près, être classée qu'avec de sérieuses réserves dans le droit public aux côtés de la doctrine administrative, la science du droit constitutionnel et la science du droit administratif sont considérées depuis longtemps comme deux disciplines étroitement imbriquées, et s'associant en un "pilier » commun pour former une communauté de discours scientifique, largement intégrée, dans le champ de la recherche comme de l'enseignement. Qu'il s'agisse du choix du sujet de la thèse ou de l'habilitation d'un "publiciste", qu'il soit question de l'habilitation à enseigner ou de l'intitulé d'une chaire universitaire, ou bien encore que le sujet soit celui des lieux et du format des échanges scientifiques, dans tous les cas, la doctrine constitutionnelle et la doctrine du droit administratif sont fondamentalement considérées et traitées comme deux branches du droit public liées entre elles. L'attente à laquelle doit satisfaire un "publiciste » est qu'il obtienne une double qualification professionnelle, à la fois en droit constitutionnel et en droit administratif. Il n'est donc guère étonnant qu'en Allemagne, la constitutionnalisation de l'ordre juridique, dont il sera encore question ultérieurement (cf. infra, IV.3.), ait trouvé son point de départ dans le domaine du droit administratif (et de la science administrative) et se soit concrétisée de la manière la plus poussée dans ce domaine. Les deux disciplines - la doctrine constitutionnelle d'une part et la doctrine du droit administratif d'autre part - possèdent un « air de famille " manifeste, du point de vue de l'approche disciplinaire ou méthodologique, ce qui ne doit toutefois pas conduire à sous-estimer ou à négliger les différences qui existent bel et bien, tant en ce qui concerne l'orientation que l'approche de ces deux disciplines ${ }^{16}$. 


\section{La symbiose entre la science du droit constitutionnel et la jurisprudence constitutionnelle}

\section{La dogmatique confrontée à la justice constitutionnelle}

15 La science juridique allemande en général est connue - certains diront : tristement célèbre - pour tirer ses repères du droit constitutionnel; et la science du droit constitutionnel en particulier est connue pour les concepts de dogmatique constitutionnelle qu'elle élabore, concepts tout autant amples et destinés à couvrir tous les domaines que finement ciselés et nuancés. Ce statut particulier - tant au niveau national qu'à l'échelon international - de la doctrine constitutionnelle en Allemagne ne résulte cependant pas exclusivement, ni même principalement, du génie propre de cette science ou de ses propres mérites. Il résulte plutôt pour l'essentiel de la combinaison de trois facteurs que la doctrine constitutionnelle n'a certes pas créés, mais dont elle a vigoureusement soutenu l'épanouissement et la synergie.

16 Ces facteurs coïncidents étaient : (1) l'entrée en vigueur d'une nouvelle constitution (la Loi fondamentale) conçue pour imposer définitivement l'état de droit, les droits fondamentaux et la justice constitutionnelle; (2) la mise en place d'une juridiction constitutionnelle (la Cour constitutionnelle fédérale) inédite par sa conception et ses compétences et qui ne tarda pas à réaliser, à développer et à mettre vigoureusement en œuvre les possibilités qui résidaient dans son statut de juridiction opérant un contrôle concret des normes et un examen de recours constitutionnels dirigés contre des décisions de justice; et enfin (3) la dogmatique en tant qu'outil scientifique dont la Cour constitutionnelle a su se servir, d'une manière inédite jusqu'alors, que ce soit pour s'y rallier ou prendre ses distances, afin d'étendre et de renforcer l'efficacité de la Constitution. Sous l'empire de la Loi fondamentale, une juridiction constitutionnelle n'hésitant pas à se positionner et à prendre des décisions et prête à imposer ces décisions trouve ainsi dans la dogmatique - prédominante depuis longtemps dans la science comme dans la pratique juridiques en Allemagne (cf. supra, II.) - un instrument qui devait lui permettre de donner au droit qui est le fondement de ses décisions - la Constitution - une vigueur qualitative et quantitative inconnue jusqu'alors ${ }^{17}$. Le raisonnement prenant la dogmatique comme point de départ - un mode de pensée qui s'était sur le plan du principe déjà imposé avant l'entrée en vigueur de la Loi fondamentale - a entamé une relation féconde avec le mode de pensée - à l'époque nouveau et n'ayant pas encore fait ses preuves - centré sur la constitution. À cette évolution a largement contribué le fait que, dans les motifs de ses décisions, la Cour constitutionnelle fédérale adopte - et tout porte à penser que le phénomène s'accentuera - un style de rédaction et un raisonnement clairement dogmatiques et scientifiques. La Cour constitutionnelle fédérale a recours à la dogmatique dans une mesure moindre que les cours suprêmes "ordinaires" des ordres de juridictions judiciaire et administrative soucieuses de préserver l'unité de la jurisprudence et de faire évoluer le droit sur certains points. Elle utilise au contraire la dogmatique d'une manière qui n'est pas sans rappeler celle de la doctrine juridique, davantage attachée à la préservation et/ou la réorientation du système dans son ensemble qu'au cas concret. La structure de la motivation des décisions de la Cour constitutionnelle fédérale, dont l'élément central est la partie consacrée aux normes de référence du contrôle de constitutionnalité, en témoigne : régulièrement, le trait de plume, la forme et le 
contenu ne diffèrent que peu, voire pas du tout, de ceux d'une contribution scientifique à un manuel de droit ou à un code commenté18. Il est tout à fait possible que cet aspect scientifique des arrêts et décisions de la Cour de Karlsruhe ait été favorisé par le fait que, parmi les membres ayant influencé la jurisprudence dans ses deux Chambres, il y a toujours eu - actuellement même de manière prononcée - des professeurs de droit public.

17 La science du droit public qui, contrairement à ce qui fut le cas sous la République de Weimar, a témoigné dès le départ de sa loyauté - aujourd'hui unanime et sans réserve envers la Constitution agit globalement comme l'alliée naturelle de la jurisprudence constitutionnelle de Karlsruhe. Ce constat ne saurait surprendre, étant donné qu'à côté de la Cour constitutionnelle fédérale, la science du droit public a été le principal bénéficiaire du changement d'attitude intervenu peu à peu depuis l'après-guerre et consistant à faire de la Loi fondamentale le point de départ du raisonnement juridique. Le fait que la science juridique utilise elle aussi la Constitution comme fondement de ses raisonnements récompense la science du droit public par un accroissement sensible de l'importance, de la portée et de la réputation de celle-ci au sein des autres disciplines juridiques - il fait même d'elle la discipline juridique reine. La doctrine constitutionnelle, à la différence, dans les années 1950, des autres organes constitutionnels et des juridictions non constitutionnelles, ne s'est d'ailleurs jamais fondamentalement ni ouvertement opposée à l'exigence de la Cour constitutionnelle fédérale de jouer un rôle de premier plan ${ }^{19}$. Bien au contraire, la doctrine juridique s'est montrée dès le départ comme un compagnon certes critique mais toujours loyal et dans les limites qui sont celles d'une discipline scientifique - un partenaire fort de la Cour constitutionnelle. Si une critique peut être adressée à la majorité de la communauté des constitutionnalistes, ce n'est pas celle d'une défiance injustifiée envers la Cour ni une critique trop exacerbée de son activité, mais bien au contraire une attitude parfois trop complaisante et prenant la forme de ce que l'on pourrait appeler un « positivisme juridictionnel » axé sur la Cour constitutionnelle fédérale ${ }^{20}$.

\section{La juridicisation de la Constitution}

Dès le début de son activité juridictionnelle en septembre 1951, la Cour constitutionnelle fédérale n'a laissé aucun doute quant à son intention de lire et d'interpréter la Loi fondamentale, de l'utiliser et de la rendre effective à un point jamais atteint auparavant par aucune autre constitution. Ce sont en particulier les différentes procédures de contrôle de constitutionnalité (contrôle des normes abstrait et concret dans le cadre d'un recours au principal ; recours constitutionnel direct dans le cadre d'un contrôle principal ou incident des normes) - procédures qui ont d'ailleurs apporté une réponse à la question autrefois épineuse de la primauté par rapport au législateur - qui ont encouragé la Cour de Karlsruhe à traiter, du point de vue de la structure et de la méthode, les dispositions de la Loi fondamentale, sans préjudice de leur statut et de leur rang particuliers parmi les normes juridiques, comme des normes juridiques comme toutes les autres et à les interpréter et à les appliquer de la même manière que ces dernières - c'est-à-dire dans le cadre et avec les outils de la méthodologie et de la dogmatique juridiques. Le fait que le droit constitutionnel soit d'une certaine manière un « droit politique ${ }^{21} »$ n'a pas conduit la Cour constitutionnelle fédérale à développer, sur le modèle de la "political question doctrine», une jurisprudence entérinant l'existence d'actes de gouvernement limitant la compétence 
de contrôle de la juridiction constitutionnelle. La doctrine constitutionnelle se rallie à cette position de la Cour constitutionnelle.

Les droits fondamentaux ont acquis très vite une importance particulière, alors que jusque-là, ces derniers n'avaient joué le rôle de normes de référence d'un contrôle sérieux de constitutionnalité - y compris de la loi - qu'au mieux dans la jurisprudence de la Cour suprême des États-Unis. Avec la collaboration vigoureuse de la doctrine constitutionnelle qui, selon les cas, fournit des travaux en amont ou en aval des décisions de la Cour et les accompagne de ses commentaires, la Cour constitutionnelle fédérale a, depuis la fin des années 1950, érigé les droits fondamentaux en un système à plusieurs dimensions, dynamique, flexible et couvrant en principe toutes les branches du droit. Les effets de synergie libérés par la rencontre entre la justice constitutionnelle et la dogmatique (constitutionnelle) se manifestent le plus clairement et le plus durablement dans le domaine des droits fondamentaux. Les garanties fondamentales offertes par ces droits - dont la structure et la teneur sont d'origine classique et tirées de contextes et de traditions antérieurs à la Loi fondamentale et donc non créées par la constitution allemande mais reprises par celle-ci - sont assemblées par la jurisprudence et la doctrine constitutionnelles pour former un ensemble à plusieurs dimensions, complet, tourné vers la résolution de conflits et ouvert sur l'avenir.

Ce système est dominé par deux idées fondamentales faisant référence l'une à l'autre et entre lesquelles existe en même temps un équilibre : d'une part, l'ambition de procurer la plus grande efficacité possible aux droits fondamentaux, d'autre part la réalisation des différents droits selon le principe de proportionnalité. Tandis que la première de ces idées directrices porte en elle une tendance à l'interprétation et à l'application des droits fondamentaux extensives et largement libérées des contingences du cas concret, la seconde sert de moyen de calibration de la protection efficace des droits fondamentaux, tant au sein de l'ordre juridique dans son ensemble que, et surtout, dans les cas concrets. Les éléments de dogmatique servant pour la réalisation de ces deux axiomes de base tracent en principe le cadre dans lequel la réflexion portant sur l'interprétation de la constitution doit s'effectuer et décrivent en même temps les modèles d'argumentation en présence et la panoplie d'arguments disponibles. Quel que soit le droit fondamental dont il est question, la Cour constitutionnelle examine un recours invoquant sa violation généralement selon un schéma en trois temps: détermination $\mathrm{du}$ domaine protégé par le droit fondamental en question, détermination de l'ingérence étatique dans ce droit, puis justification possible de cette ingérence. Le fait que le texte de la constitution lui-même soit muet sur ce schéma tripartite "domaine protégé/ingérence/limites du droit fondamental » n'est de nos jours même plus mentionné, ni ne donne lieu à aucune réflexion particulière. Un élément supplémentaire pour l'édification de ce système réside dans le raisonnement selon lequel les droits fondamentaux constituent d'une part des garanties de domaines particuliers, mais prévoient aussi, d'autre part, des garanties générales et «fourretout", applicables quand aucun droit fondamental spécial ne s'applique. Cette constatation vaut pour les libertés fondamentales comme pour les garanties du principe d'égalité. L'ingéniosité propre à ce mode de raisonnement est qu'il assure une garantie en principe sans lacunes des droits fondamentaux. La pensée systématique pousse à l'établissement d'un système sans faille et ne peut concevoir l'idée selon laquelle la protection des droits fondamentaux pourrait n'être assurée que ponctuellement ou de manière sectorielle. Dans la lignée de cette tendance à 
l'extension de la portée des droits fondamentaux, la Cour considère que toute "situation particulière mettant en danger des droits fondamentaux" (grundrechtstypische Gefährdungslage) 22 dans laquelle se trouve le citoyen vis-à-vis de l'État, et susceptible de déclencher une protection du premier contre le second, est plus importante que le libellé de la définition du droit fondamental touché tel qu'il se trouve dans le texte de la Constitution. La conséquence de cette jurisprudence est qu'il n'est pas difficile de découvrir par voie d'interprétation de la Constitution de nouvelles garanties offertes par les droits fondamentaux existants si survient une situation nouvelle de mise en danger de ces droits. L'extraordinaire multiplication des droits fondamentaux sous l'empire de la Loi fondamentale trouve l'un de ses principaux catalyseurs dans la découverte du caractère multidimensionnel des garanties offertes par les droits fondamentaux. L'extension des droits fondamentaux - ou plutôt l'interprétation extensive du domaine protégé par les droits fondamentaux s'accompagne toutefois d'une tendance à maints égards parallèle - l'on pourrait aussi dire une tendance complémentaire - du côté des limites des droits fondamentaux. Dans la mesure où la protection conférée par ces droits est entendue dans un sens généralisé et libéré des bornes de son champ d'application, surgit en effet la nécessité systémique - d'interpréter de façon extensive également les possibilités de restriction des droits fondamentaux et de les actualiser. La jurisprudence des juges de Karlsruhe satisfait à cette exigence lorsqu'elle nivelle - sinon expressément, du moins sur le fond - le régime des restrictions différencié prévu dans le texte de la Loi fondamentale et qu'elle le soumet à un arbitrage entre les différents intérêts en présence. Le principe de proportionnalité, idée centrale pour toute justification de l'ingérence dans un droit fondamental et initialement développé par la Cour au sujet des ingérences dans les libertés publiques, rayonne désormais également sur la structure et la dogmatique relatives au principe d'égalité. Ce système ouvert et dynamique de la dogmatique de la Cour constitutionnelle en matière de droits fondamentaux permet, d'une part, notamment à travers une homogénéisation des garanties offertes par les droits fondamentaux, de désigner et de traiter pratiquement chaque question de droit comme une question touchant aux droits fondamentaux. D'autre part, le recours - pour des raisons systémiques, tout aussi généralisé - au principe de proportionnalité ouvre la possibilité de piloter de manière différenciée tant la protection efficace des droits fondamentaux dans un cas concret que l'étendue de la protection juridictionnelle assurée par la Cour constitutionnelle. Ce qui rend possibles cette forme particulière de développement de la Constitution sans révision formelle de son texte et cette flexibilité qui permet de régler un cas particulier sans s'engager à l'avance à traiter les cas futurs avec la même indulgence, c'est la dogmatique.

\section{La constitutionnalisation de l'ordre juridique et de la pensée juridique}

21 La Loi fondamentale apparaît de plus en plus comme un code source de l'ordre juridique. La découverte de l'idée que "la Constitution peut s'appliquer à tout ${ }^{23}$ ", un principe que la Cour constitutionnelle fédérale a désigné dans son arrêt « Lüth » sous le nom de "effet de rayonnement " des droits fondamentaux, conduit d'une part à ce qu'aucune disposition de droit ordinaire ne puisse plus être interprétée et appliquée d'une manière qui serait contraire à la Loi fondamentale et aux exigences que cette dernière contient et d'autre part à ce que toute disposition de droit ordinaire doive être 
lue à la lumière de l'esprit de la Constitution ${ }^{24}$. Plus la teneur d'une disposition constitutionnelle est généralisée et dense - teneur dont la signification exacte a été dégagée par la dogmatique -, plus les exigences seront étendues auxquelles les normes de valeur infra-constitutionnelle devront satisfaire. Dès que et dans la mesure où des droits fondamentaux sont en jeu - et tel est régulièrement le cas du système des droits fondamentaux tel qu'il a été formulé (cf. supra) à l'aide de la dogmatique se concentrant sur l'aspect matériel -, il n'est plus possible pour le juge de dire le droit sans tenir compte de la Constitution.

22 L'évolution, connue sous le terme de constitutionnalisation, consistant pour le juge à s'orienter de plus en plus vers les principes de la Constitution lorsqu'il interprète le droit ordinaire ${ }^{25}$ repose, outre sur la conception systématiquement étendue des droits fondamentaux et guidée par l'idée d'une optimisation de la portée de ces droits, sur deux piliers supplémentaires, à savoir la procédure du recours constitutionnel dirigé contre une décision de justice et l'interprétation conforme d'une norme à la Constitution. Les recours constitutionnels directs, lorsqu'ils sont dirigés contre une décision de justice, fournissent - déjà en raison de leur nombre très élevé : près de 190000 recours de ce type en près de 60 ans - à la Cour constitutionnelle fédérale un instrument qui lui permet d'assurer un contrôle efficace sur la manière dont les juridictions ordinaires s'acquittent concrètement de leur obligation de tenir compte des droits fondamentaux. Cet instrument permet en outre aux gardiens des droits fondamentaux de Karlsruhe de préciser la portée des droits fondamentaux jusque dans les moindres détails d'un cas concret et individuel. À cet égard, une importance primordiale et souvent décrite revient au principe de proportionnalité : par son objet et son utilisation, ce dernier empêche que la procédure du recours constitutionnel contestant une décision de justice puisse un jour devenir superflue, au motif que les tribunaux ordinaires auraient pour chaque cas concret correctement prédit la portée des droits fondamentaux en jeu.

23 Quant à ce que l'on appelle "l'interprétation conforme ", c'est-à-dire la réinterprétation $\mathrm{du}$ droit non-constitutionnel (prétendument) induite par la Constitution, elle n'est pas nécessairement liée à une intervention de la part de la Cour constitutionnelle fédérale, mais se rattache au fait que le juge est lié par la Constitution $^{26}$. Bien que l'objection selon laquelle cette technique ne constituerait pas simplement une forme particulière d'interprétation de la Constitution destinée à assurer la primauté de cette dernière, mais une manière "froide », et allant au-delà de la décision de justice, de censurer partiellement une norme (sans amputation de parties du texte de la norme) ne puisse être réellement rejetée, la majorité de la doctrine publiciste se rallie à la position de la Cour constitutionnelle fédérale et prône l'idée que la technique de "l'interprétation conforme" est la manifestation d'un "principe servant à maintenir une norme, un principe que connaît tout ordre construit sous forme de hiérarchie ${ }^{27} »$. Cette technique a elle aussi pour effet de renforcer la compatibilité du droit infra-constitutionnel avec la Constitution et l'orientation croissante de ce droit vers la Constitution.

24 L'exemple de la théorie des principes formulée par Robert Alexy illustre la constitutionnalisation de la pensée juridique. Alors qu'à l'échelon mondial, les avantages et les inconvénients de ce modèle font l'objet de débats de théorie du droit menés par des théoriciens du droit - débats de plus en plus internationalisés -, l'idée avancée par Alexy et qu'il avait initialement formulée comme le fondement de sa 
"Théorie des droits fondamentaux ${ }^{28}$ » est reprise ou rejetée en Allemagne surtout par les publicistes en leur qualité de membres de la doctrine constitutionnelle. La différence du cadre des débats - la théorie du droit d'une part, la dogmatique constitutionnelle (et/ou la théorie de la constitution) d'autre part - ne manque logiquement pas d'influer considérablement sur la forme et le contenu de ces controverses.

25 La constitutionnalisation engendre toutefois encore un autre effet qui, d'une certaine manière, va dans la direction inverse. Dans la mesure où de nouveaux champs sont ouverts à l'application de la Constitution, avec la Cour constitutionnelle fédérale comme gardienne de cette dernière, les compétences de la science du droit constitutionnel s'élargissent également et « contraignent » celle-ci à se préoccuper de cas relevant du droit ordinaire.

\section{La « dogmatisation » de la pensée constitutionnelle}

"L'immense force créatrice de la jurisprudence de la Cour constitutionnelle fédérale ${ }^{29}$ " a pour effet de faire participer la science constitutionnelle au renforcement du droit constitutionnel et de la dogmatique constitutionnelle. Mais elle a aussi un prix, que l'on pourrait désigner par le terme de « dogmatisation » de la pensée sur la constitution. La science du droit public a fort à faire pour suivre, développer et encadrer la dogmatique constitutionnelle tracée par la Cour constitutionnelle. Si les membres de la doctrine de droit public veulent être en mesure d'exercer une influence sur cette dogmatique constitutionnelle jurisprudentielle, ils doivent exposer leurs doutes et leurs suggestions, leurs idées et leurs arguments en employant le langage de la dogmatique. En Allemagne, même la théorie constitutionnelle, qui, par sa nature, est plutôt éloignée de la pratique, garde un contact plus ou moins étroit avec la dogmatique constitutionnelle. Contrairement à ce qui se passe dans des pays comme la France ou les États-Unis, qui ne connaissent pas de discipline juridique comparable à celle de la dogmatique allemande, la théorie constitutionnelle développée en Allemagne entretient des liens plus étroits avec la dogmatique. Cette situation lui assure certes la possibilité d'exercer une plus grande influence sur la pratique du droit, mais elle doit en payer le prix, en ce sens que, globalement, cette proximité aboutit à une réduction sensible - induite par la dogmatique - du champ de discussion ${ }^{30}$. Les modèles ou les approches théoriques qui s'écartent de la jurisprudence et la dogmatique formulées à Karlsruhe, voire qui s'opposent fondamentalement à elles, n'ont que des chances réduites d'attirer l'attention et d'obtenir l'adhésion dans les débats de la doctrine de droit public.

27 La capacité d'innovation de la théorie constitutionnelle et l'influence de la pratique de la Constitution sont d'une certaine manière comme des vases communicants : plus l'une augmente, plus l'autre diminue, et vice versa. Alors que la doctrine de droit public sous la République de Weimar, par son attitude ambiguë vis-à-vis de la Constitution de Weimar et ses querelles sur la direction à suivre, n'a que peu pesé sur la pratique du droit, la science du droit public développée sous l'empire de la Loi fondamentale dans le sillage de la jurisprudence de la Cour constitutionnelle fédérale ne saurait se plaindre d'un manque de pertinence pour la pratique du droit, y compris dans le cadre du dialogue entre la doctrine et la Cour constitutionnelle. 


\section{L'attitude de la Loi fondamentale à l'égard de l'Europe : un point de rupture?}

Tout semble laisser à penser que la relation quasi-symbiotique entre la Cour constitutionnelle fédérale et la doctrine de droit public se voit confrontée à un défi sérieux lorsqu'il s'agit de déterminer la position au sein de l'Union européenne de l'État de droit mis en place par la Loi fondamentale. À cet égard, il apparaît surtout ces derniers temps que les anciens rapports et liens de loyauté ne sont pas forcément adaptés aux conditions de l'avenir. En conséquence, le débat tant au sein de la Cour constitutionnelle ${ }^{31}$ que parmi la doctrine constitutionnelle est particulièrement vif. L'enjeu de ce débat n'est pas "seulement » de trouver une réponse constitutionnelle face à "l'Europe", mais également de déterminer (1) la place de la Cour constitutionnelle fédérale au sein d'une "fédération européenne des juridictions constitutionnelles ${ }^{32} \|^{33}$, (2) l'orientation fondamentale et l'identité de la doctrine allemande de droit public, ainsi qu'en dernier lieu (3) la relation entre ces deux dimensions, ce qui explique la gravité et les préoccupations du discours, de part et d'autre. Il serait certes prématuré de voir approcher la fin de cette communauté unique basée sur la fécondation réciproque et ayant connu un tel succès en Allemagne, mais l'on doit accepter que l'on s'interroge, au sein de la science du droit constitutionnel, sur les effets que le recul de la domination de la jurisprudence de la Cour constitutionnelle fédérale peut produire sur la doctrine du droit public, sur les plans thématique, méthodologique et disciplinaire ${ }^{34}$.

\section{La science du droit administratif : entre " dépendance de la Constitution » et « orientation extérieure »}

29 Tandis que le cadre de réflexion de la doctrine constitutionnelle allemande est pour l'essentiel tracé par la jurisprudence des juges constitutionnels de Karlsruhe, on peut dire, en simplifiant, que la doctrine administrative - liée à la doctrine constitutionnelle dans le cadre de la communauté scientifique de droit public où sont menés les débats (cf. supra, III.) - oscille entre "dépendance de la Constitution ${ }^{35}$ " et "orientation extérieure $^{36} »$.

\section{La constitutionnalisation du droit administratif et de la science administrative}

C'est sur le droit administratif, et avec lui sur la science administrative, que l'accent mis sur la Constitution a produit ses effets en premier, de manière la plus complète et le plus durablement. Même avant que la Cour constitutionnelle fédérale n'y ait apporté sa contribution, la Cour fédérale administrative et la doctrine de droit public ont montré et attiré l'attention sur le fait que le droit administratif n'était pas autre chose que du «droit constitutionnel concrétisé ${ }^{37} \rrbracket^{38}$.

Pour l'essentiel, c'est la combinaison du droit subjectif - dont les droits fondamentaux constituent le paradigme - et de la protection juridique - qui est depuis toujours la principale incarnation en Allemagne de l'idée de l'État de droit - qui forme le duo 
fondamental constitutionnalisant le droit administratif et la science du droit administratif. C'est de ce double mouvement de pensée, guidé d'une part par le droit subjectif et d'autre part par la protection juridique (de l'individu), que sont nés le droit administratif et la science administrative d'après-guerre en Allemagne. C'est sous son influence que "tombe " le principe du «rapport d'autorité particulier», qui avait auparavant dominé le droit public et conféré à celui-ci le caractère d'un droit basé sur un rapport de subordination, un droit spécial au bénéfice de l'État; en vertu du droit à une protection juridictionnelle efficace garantie par l'article 19, alinéa 4 , de la Loi fondamentale, le caractère de la forme de l'action de l'État, jadis déterminante pour le déclenchement de la protection juridique, est relégué au second plan; le principe de la légalité est élargi significativement et s'étend, sous l'effet de la théorie des garanties essentielles (Wesentlichkeitstheorie) jusque dans le domaine des services publics (Leistungsverwaltung); les modalités des procédures et de l'organisation administratives sont remises en question sous l'angle des effets pour le renforcement des droits fondamentaux qu'elles sont susceptibles d'engendrer; une attention particulière est désormais accordée aux rapports juridiques pluripartites, en tant que situations subjectivisées concernant la protection de tiers; les dispositions administratives, conçues comme des règles internes flexibles et malléables, entrent, du fait du "principe de l'autolimitation de l'administration », dans le champ du principe d'égalité (article 3, alinéa $1^{\mathrm{er}}$, de la Loi fondamentale) et tombent donc sous l'influence de ce dernier; le pouvoir discrétionnaire de l'administration et ses marges d'appréciation sont, sous l'aspect de la protection juridique (intensité du contrôle opéré) et des droits fondamentaux, soumis à un régime plus exigeant quant à leur justification; anobli par son application en droit constitutionnel et en particulier dans le domaine des droits fondamentaux, le principe de proportionnalité, initialement développé dans le domaine du droit de la police administrative et « exporté » depuis vers le droit constitutionnel, déploie une efficacité ubiquitaire. Il est en principe applicable lors de toute interprétation du droit («interprétation conforme à la Constitution»!) et de toute application du droit par l'administration, laquelle est liée par les droits fondamentaux. La conséquence en est une «juridicisation et une soumission aux tribunaux croissantes » de l'action de l'administration ${ }^{39}$ - et en conséquence un repositionnement de la science du droit administratif. Dans ce contexte, un rôle central revient au droit administratif général - façonné d'abord par la science du droit administratif, puis ensuite par la jurisprudence des juridictions administratives et oscillant étrangement entre droit positif et science du droit -, car ce droit assure la fonction déterminante "d'intermédiaire entre le droit constitutionnel et le droit administratif spécial ${ }^{40}$ ». Ce constat est d'autant plus remarquable que le droit administratif général est considéré comme l'incarnation par excellence de la pensée dogmatique systémique en droit public (et dans la science du droit public) ${ }^{41}$. Une fois de plus, la dogmatique axée sur la notion de système démontre son efficacité, notamment à travers l'effort pour rendre compatible le droit constitutionnel et le droit infra-constitutionnel.

\section{Réorientations et tendances à l'ouverture}

32 La constitutionnalisation n'est pas le seul modèle du droit administratif et de la doctrine du droit administratif dans l'Allemagne de l'après-guerre. Ces dernières décennies, deux évolutions - à maints égards entrelacées - poussent le droit administratif et plus encore la doctrine administrative à se réorienter et, du coup, à 
relativiser d'une certaine manière ou du moins à enrichir l'accent mis sur le droit constitutionnel. Ces évolutions ont toutes deux leurs origines à l'extérieur de la Constitution. La première, à savoir l'européanisation (et, dans un degré moindre, l'internationalisation), résulte pour l'essentiel des effets produits par le droit de l'Union européenne (auparavant le droit communautaire), lequel prime en principe sur toute norme nationale des États membres et dispose, avec le principe de "l'effet utile", d'une clef passe-partout pour ouvrir aux effets du droit de l'Union toute disposition juridique nationale. La Cour constitutionnelle fédérale et, avec elle, la science du droit constitutionnel font largement figure d'observatrices extérieures dans cette évolution, étant donné que les dispositions du droit de l'Union ne constituent pas des normes de référence pour le contrôle de constitutionnalité opéré par les juges de Karlsruhe. Dès lors, l'européanisation du droit administratif allemand s'effectue en majeure partie en dehors du droit constitutionnel et de l'intervention de la Cour constitutionnelle fédérale. De ce fait, l'européanisation du droit administratif se superpose partiellement (mais de manière croissante) à la constitutionnalisation de ce droit.

33 La seconde évolution, qu'il n'est pas aisé de résumer au moyen d'un concept homogène, trouve ses origines dans la tentative pour formuler une science du droit administratif moderne, répondant aux réalités nouvelles de la vie administrative comme aux connaissances modernes dégagées par les disciplines non juridiques et (davantage) susceptible d'intéresser d'autres disciplines. Renforcée par la « discussion sur la réforme » notamment pendant les années $1990^{42}$, cette évolution s'est manifestée dans le projet d'envergure et programmatique de formuler une « Nouvelle science du droit administratif ${ }^{43}$ ». Le nouveau modèle est celui du pilotage. Il oriente et produit une multitude de mouvements de recherche et d'ouvertures jusqu'alors méconnus - en visant dans un premier temps le monde réel de l'administration, mais ensuite également une orientation de la discipline et des méthodes. Dès l'origine, il reflète dans son ambition globale l'esprit de la dogmatique fondée sur un système de pensée; malgré son effort d'ouverture, il n'abandonne pas le projet, plutôt tourné vers l'établissement d'un système fermé, d'une dogmatique administrative, mais au contraire donne une nouvelle dimension à l'idée de système inhérente à la dogmatique et selon laquelle les connaissances sont maitrisables avec les moyens de la science.

$34 \mathrm{Du}$ point de vue disciplinaire et méthodologique, la «Nouvelle science du droit administratif » présente un visage hybride. Il n'est pas faux de constater que - avec son ambition holistique consistant à vouloir prendre en compte l'intégralité des effets de pilotage pour les soumettre à la science du droit - la « Nouvelle science du droit administratif» contribuerait à rendre confuses les «imputations normatives de compétences et de responsabilités ${ }^{44} »$. Plus l'imbrication au sens strict entre perspectives juridiques et non-juridiques est forte, moins la teneur juridique des énoncés ainsi produits est identifiable ${ }^{45}$; mais d'un autre côté, ces derniers sont alors plus susceptibles de faire l'objet de débats menés ailleurs (que cet « ailleurs » renvoie à une discipline scientifique ou à un lieu) et n'étant pas aussi stricts en ce qui concerne les aspects liés à la discipline et aux méthodes. Il est même permis d'aller encore plus loin et d'affirmer que du fait de la diversité des perspectives - un élément constitutif de toute science du droit administratif qui se veut informée de manière interdisciplinaire - et de l'utilisation de notions employées par plusieurs disciplines - alors que ces notions n'ont, au mieux, que partiellement été revêtues d'un contenu juridique - la nouvelle approche en termes de pilotage dépasse de plus en plus le rôle et le cadre de la 
dogmatique classique. Alors que cette dernière vise, dans le débat intra-juridique, à fournir une explication pour l'application du droit - explication fiable, standardisée et maintenue à une dimension (réduite) permettant aux acteurs juridiques de la traiter -, l'ambition de la «Nouvelle science du droit administratif » est justement de mener dans la perspective d'une science administrative moderne un discours de pilotage ambitieux en matière de trans- et d'interdisciplinarité. Pour la science du droit administratif, le prix à payer de cette capacité à générer des liens trans- ou interdisciplinaires est, semble-t-il, qu'elle perd en pertinence pour la pratique du droit. Le fait qu'au moins une partie des membres de la science du droit administratif estiment acceptable de relativiser ainsi le caractère dogmatique et la capacité des idées et modèles de cette science à être repris par la jurisprudence est, sans doute, dû également au fait que le lien entre science administrative et jurisprudence administrative est traditionnellement moins étroit et moins généralisé que celui qui unit la doctrine constitutionnelle et la jurisprudence constitutionnelle. L'affinité de la Cour fédérale administrative pour la science du droit, nettement plus réduite, que celle de la Cour constitutionnelle fédérale, se manifeste déjà dans la structure et le style de rédaction - correspondant typiquement à ceux d'une juridiction de cassation - des motifs de ses décisions et arrêts ${ }^{46}$. Cette moindre affinité apparaît de manière particulièrement claire lorsqu'il y a un décalage entre l'état de la dogmatique scientifique et celui de la dogmatique de la Cour, comme ce fut longtemps le cas sur la question de la reconnaissance et du respect des effets du droit communautaire (désormais droit de l'Union) par le droit (administratif) allemand.

35 Du point de vue de l'assouplissement ou de l'affaiblissement du caractère dogmatique (systématicité), la boucle est bouclée par rapport à la première évolution mentionnée, à savoir l'européanisation du droit administratif : la jurisprudence de la Cour de justice et du Tribunal de l'Union européenne se nourrit de traditions juridictionnelles diverses, parmi lesquelles on trouve des systèmes juridiques fondés sur la règle du précédent, mais aussi des traditions auxquelles la culture de la motivation ample, comme en Allemagne, est étrangère. Cette situation conduit à ce qu'une dogmatisation habituelle en Allemagne vis-à-vis des juridictions nationales - apparaisse, eu égard à la jurisprudence des juridictions de l'Union, largement inappropriée, pour ne pas dire déplacée. Cet élément oblige lui aussi la science administrative allemande européanisée soit à élargir sa conception d'une dogmatique centrée sur la notion de système, soit à la modifier dans son ensemble ou du moins à s'accommoder d'un degré moindre de dogmatisation et de systématisation.

\section{La science du droit public dans le cadre de la science européenne commune du droit public : un bref aperçu}

\section{La contribution allemande lors de la formation de la science européenne commune du droit public}

Jusqu'à présent, la doctrine de droit public allemande s'est globalement montrée ouverte aux multiples dimensions de l'européanisation et a activement contribué à cette dernière. Elle peut même prétendre avoir fourni des impulsions majeures et des contributions exceptionnelles pour l'établissement d'une doctrine commune 
européenne de droit public : à cet égard, il convient de citer la fondation, à l'initiative de l'Association des professeurs allemands de droit public, d'une association européenne des professeurs de droit public, la Societas Iuris Publici Europaei (SIPE), en $2003^{47}$, ou encore l'ouvrage classique Ius Publicum Europaeum (IPE), publié en majorité sous égide allemande et comportant des contributions en langue allemande de juristes d'environ une douzaine des États membres de l'Union européenne, ainsi que de la Suisse ${ }^{48}$. En ce qui concerne d'une part l'impact du droit européen sur les ordres juridiques nationaux et les sciences juridiques nationales, et d'autre part l'édification d'une science européenne commune, la science allemande du droit public n'a pas à rougir.

Cette contribution allemande dans le cadre de l'émergence et de la formation du discours européen commun sur le droit est d'autant plus importante et urgente que la manière de pratiquer en Allemagne la science du droit en général, et plus particulièrement du droit public, constitue à maints égards, si l'on considère les ordres juridiques, les sciences juridiques et les cultures juridiques des États membres de l'Union européenne, une forme "d'exception allemande ", ou du moins d'exception limitée aux pays germanophones. Si «l'approche allemande » n'est pas expliquée à temps, durablement, clairement et par des voies multiples, s'il n'est pas fait d'efforts pour vanter les mérites de cette approche, alors le courant principal du débat européen s'engouffrera dans d'autres voies. Évidemment, il ne s'agit pas là de prôner un «nationalisme méthodologique " visant à préserver ce qui est familier à soi-même, mais, en parallèle, il faut aussi se garder de l'autre extrême, c'est-à-dire la fuite dans une " euphorie de la convergence ${ }^{49}$ ». Il convient en outre de souligner que l'objectif n'est pas simplement de remplacer des débats nationaux de la science du droit par un unique discours européen commun sur le droit, mais au contraire de faire coexister ces deux échelons de discours dans un rapport dialectique - ou pour reprendre le crédo ecclésiologique du $\mathrm{II}^{\mathrm{e}}$ Concile œcuménique du $\operatorname{Vatican}^{50}:$ « in quibus et ex quibus ».

\section{Dépendances au sentier de la doctrine de droit public, obstacles à son internationalisation}

Ce projet doit faire face à des difficultés diverses - indépendamment même de la véritable question, qui est de savoir quels aspects doivent concrètement être préservés ou transposés. Parmi ces difficultés figurent celles que l'on pourrait qualifier de dépendances au sentier faisant obstacle à l'européanisation, voire à l'internationalisation: des caractéristiques des débats allemands sur le droit qui dans l'une et/ou l'autre direction (c'est-à-dire de l'intérieur vers l'extérieur et/ou de l'extérieur vers l'intérieur) se présentent comme autant d'obstacles à ce qu'elles puissent être reprises à l'échelon européen ou international.

Dans une large mesure, ces obstacles ne touchent pas la seule doctrine de droit public, mais concernent la science du droit en Allemagne dans son ensemble. On peut citer ici en premier lieu le fait que les débats à l'échelon national sont intrinsèquement liés à la langue allemande, un obstacle difficile à surmonter si l'on prend en considération que le droit, en tant que produit d'une certaine culture, dépend de manière spécifique de la langue et que la pratique du droit, à laquelle la science du droit se réfère (par exemple les lois adoptées ou encore la jurisprudence de la Cour constitutionnelle fédérale), communique en langue allemande. Un autre obstacle, d'ordre doctrinal et général, 
réside dans l'attachement de la doctrine à une perspective, des exigences et à un format de type spécifiquement dogmatique; dès lors, la reprise d'approches juridiques s'étendant bien au-delà de l'horizon de la dogmatique n'est possible que dans des limites étroites. Une autre conséquence, agissant comme un obstacle, est que la formation des juristes en Allemagne, sanctionnée par les examens d'État, lie la recherche avec l'enseignement, limitant ainsi la pertinence d'échanges internationaux pour les chercheurs comme pour les étudiants. Si l'on considère seulement les systèmes de " common law ", mais aussi le droit de l'Union européenne, où la division tripartite en droit privé, droit public et droit pénal est inconnue, la doctrine des "trois empires", qui continue d'être rigoureusement suivie en Allemagne, constitue parfois un obstacle gênant pour les débats. Eu égard au fait qu'en droit comparé, le sens de la comparaison va du plus petit au plus grand, la comparaison, en Allemagne, est la plupart du temps menée avec des ordres et des débats juridiques ayant un "poids » comparable ou plus élevé que ceux de l'Allemagne (États-Unis, France, Grande-Bretagne, Italie, Espagne l'Autriche et la Suisse, en raison de la langue, constituent des exceptions notables), alors que des ordres et des débats juridiques plus «petits » ne sont guère au centre de l'attention. Un obstacle particulier à la réception de publications scientifiques étrangères réside en Allemagne dans la concentration sur la jurisprudence de la Cour constitutionnelle fédérale. Ceci conduit à ce que la convention CEDH et la jurisprudence de la Cour européenne des droits de l'homme se trouvent pour ainsi dire rejetées dans l'ombre par les droits fondamentaux de la Loi fondamentale dans leur interprétation par la Cour constitutionnelle fédérale. En conséquence, la connaissance en Allemagne de ce niveau européen, de plus en plus important, des droits fondamentaux demeure en partie nettement plus réduite que celle qui prévaut dans d'autres pays ayant adhéré à la $\mathrm{CEDH}^{51}$ - et ce, alors que la Cour européenne des droits de l'homme s'appuie plus d'une fois sur des traditions d'interprétation et d'application des droits fondamentaux forgées à Karlsruhe. Le pays possédant la dogmatique la plus évoluée et la plus poussée en matière de droits fondamentaux appartient, lorsqu'il s'agit de la dogmatique relative aux droits de l'homme (tels que les entend la Convention EDH) plutôt aux pays en voie de développement.

\section{Tirer des enseignements de la doctrine de droit public ?}

Si l'on s'interroge en fin de compte sur l'utilité de l'apport de la doctrine de droit public allemande à une science du droit européenne commune, quatre éléments viennent particulièrement à l'esprit, et il convient de les mentionner au moins brièvement. Il s'agit en premier lieu de l'imbrication, féconde pour les deux disciplines impliquées, de la science du droit constitutionnel et de la science du droit administratif; en deuxième lieu, de la riche expérience d'interaction avec une juridiction constitutionnelle active, puissante et montrant une grande affinité pour la science ; troisièmement, de toutes les connaissances accumulées relatives au phénomène de constitutionnalisation, qui affecte tous les niveaux du droit et présente quelques ressemblances remarquables avec le phénomène d'européanisation du droit des États membres de l'Union européenne ; enfin - last but not least - de la connaissance de l'efficacité (et des limites) de cette discipline tournée vers la pratique juridique, et orientée vers l'étude du droit en vigueur, qu'est la dogmatique. 


\section{BIBLIOGRAPHIE}

Alexy, R. (1985) : Theorie der Grundrechte, Francfort-sur-le-Main, Suhrkamp.

Bachof, O. (1963) : « Über einige Entwicklungstendenzen im gegenwärtigen Deutschen Verwaltungsrecht », in : Bachof, O. (1979) : Wege zum Rechtsstaat, Königstein im Taunus, Athenäum Verlag, p. 245-262.

Becker, U. (2010) : « Rechtsdogmatik und Rechtsvergleich im Sozialrecht », in : Becker, U. (éd.) : Rechtsdogmatik und Rechtsvergleich im Sozialrecht, I, Baden-Baden, Nomos (= Studien aus dem MaxPlanck-Institut für ausländisches und internationales Sozialrecht, 49), p. 11-62.

Bogdandy, A. v. (2011) : « Deutsche Rechtswissenschaft im europäischen Rechtsraum », in : Juristenzeitung, 66 (1), p. 1-6.

Brohm, W. (2001) : « Kurzlebigkeit und Langzeitwirkung der Rechtsdogmatik », in : Geis, M.-E. / Lorenz, D. (éd.) : Staat, Kirche, Verwaltung: Festschrift für Hartmut Maurer zum 70. Geburtstag, Munich, C. H. Beck, p. 1079-1090.

Classen, C. D. (2009) : « Legitime Stärkung des Bundestages oder verfassungsrechtliches Prokrustesbett? Zum Urteil des BVerfG zum Vertrag von Lissabon », in : Juristenzeitung, 64 (18), p. 881-889.

Classen, C. D. (2010) : « Anmerkung », in : Juristenzeitung, 65 (23), p. 1186-1188.

Gärditz, K. F. / Hillgruber, C. (2009) : « Volkssouveränität und Demokratie ernst genommen Zum Lissabon-Urteil des BVerfG », in : Juristenzeitung, 64 (18), p. 872-881.

Grabenwarter, C. (2009) : Europäische Menschenrechtskonvention, $4^{\mathrm{e}}$ éd., Munich, C. H. Beck.

Häberle, P. (1992) : « Gemeineuropäisches Verfassungsrecht », in : Häberle, P. : Rechtsvergleichung im Kraftfeld des Verfassungsstaates, Berlin, Duncker \& Humblot, p. 71-104.

Hillgruber, C. (2011) : « Anmerkung », in : Juristenzeitung, 66 (22), p. 1118-1121.

Hoffmann-Riem, W. / Schmidt-Aßmann, E. (1993-2004) : « Schriften zur Reform des Verwaltungsrechts ", Baden-Baden, Nomos :

t. 1 (1993) : Reform des allgemeinen Verwaltungsrechts ;

t. 2 (1994): Innovation und Flexibilität des Verwaltungshandelns;

t. 3 (1996) : Öffentliches Recht und Privatrecht als wechselseitige Auffangordnungen;

t. 4 (1997) : Verwaltungsorganisationsrecht als Steuerungsressource ;

t. 5 (1998) : Effizienz als Herausforderung an das Verwaltungsrecht;

t. 6 (1999) : Strukturen des Europäischen Verwaltungsrechts ;

t. 7 (2000) : Verwaltungsrecht in der Informationsgesellschaft ;

t. 8 (2001): Verwaltungskontrolle;

t. 9 (2002): Verwaltungsverfahren und Verwaltungsverfahrensgesetz ;

t. 10 (2004): Methoden der Verwaltungsrechtswissenschaft.

Hoffman-Riem, W. / Schmidt-Aßmann, E. / Voßkuhle, A. (2006-2008) : Grundlagen des Verwaltungsrechts, 3 tomes, $2^{\mathrm{e}}$ éd. (2012), Munich, C. H. Beck.

Hollerbach, A. (1969) : «Ideologie und Verfassung », in : Maihofer, W. (éd.) : Ideologie und Recht, Francfort-sur-le-Main, Klostermann, p. 37-61.

Hoyningen-Huene, P. (2008) : « Systematicity: The Nature of Science », in : Philosophia Philosophical Quarterly of Israel, 36 (2), p. 167-180. 
Isensee, J. (1992) : « Verfassungsrecht als “politisches Recht” » in : Isensee, J. / Kirchhof, P. (éd.) : Handbuch des Staatsrechts der Bundesrepublik Deutschland, t. VII, $1^{\text {re }}$ éd., Heidelberg, C. F. Müller, p. 103-164.

Jestaedt, M. (2002) : «Verfassungsgerichtspositivismus. Die Ohnmacht des Verfassungsgesetzgebers im verfassungsgerichtlichen Jurisdiktionsstaat », in : Depenheuer, 0. / Heintzen, M. / Axer, P. / Jestaedt, M. (éd.) : Nomos und Ethos, Berlin, Duncker \& Humblot, p. $183-228$.

Jestaedt, M. (2010) : « Verfassungstheorie als Disziplin », in : Depenheuer, O. / Grabenwarter, C. (éd.) : Verfassungstheorie, Tübingen, Mohr Siebeck, p. 4-56.

Jestaedt, M. (2011a) : « Eine deutsche Perspektive », in : Masing, J. / Jouanjan, O. (éd.) : Verfassungsgerichtsbarkeit, Tübingen, Mohr Siebeck, p. 37-66.

Jestaedt, M. (2011b) : «Wissenschaftliches Recht - Rechtsdogmatik als gemeinsames Kommunikationsformat von Rechtswissenschaft und Rechtspraxis ", in : Jabloner, C. / KucskoStadlmayer, G. / Muzak, G. / Perthold-Stoitzner, B. / Stöger, K. (éd.) : Vom praktischen Wert der Methode. Festschrift für Heinz Mayer zum 65. Geburtstag, Vienne, Manz Verlag, p. 169-188.

Krebs, W. (2004) : « Die Juristische Methode im Verwaltungsrecht », in : Hoffmann-Riem, W. / Schmidt-Aßmann, E. (éd.) : Schriften zur Reform des Verwaltungsrechts, t. 10 : Methoden der Verwaltungsrechtswissenschaft, Baden-Baden, Nomos, p. 209-222.

Larenz, K. (1960) : Methodenehre der Rechtswissenschaft, Berlin, Springer.

Lembke, U. (2009) : Einheit aus Erkenntnis?, Berlin, Duncker \& Humblot.

Lepsius, O. (2008) : « Zur Bindungswirkung von Bundesverfassungsgerichtsentscheidungen », in : Scholz, R. / Lorenz, D. / Pestalozza, C. / Kloepfer, M. / Jarass, H. D. / Degenhart, C. / Lepsius, O. (éd.) : Realitätsprägung durch Verfassungsrecht, Berlin, Duncker \& Humblot, p. 103-118.

Lepsius, O. (2011) : « Die maßstabsetzende Gewalt », in : Jestaedt, M. / Lepsius, O. / Möllers, C. / Schönberger, C. (éd.) : Das entgrenzte Gericht, Berlin, Suhrkamp, p. 159-280.

Lerche, P. (2001) : « Stil und Methode der verfassungsrechtlichen Entscheidungspraxis », in : Badura, P. / Dreier, H. (éd.) : Festschrift 50 Jahre Bundesverfassungsgericht, t. 1, Tübingen, Mohr Siebeck, p. 333-361.

Löwer, W. (2005) : «Zuständigkeit und Verfahren des Bundesverfassungsgerichts », in : Isensee,

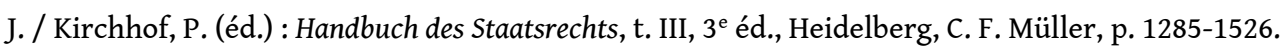

Möllers, C. (2011) : « Legalität, Legitimität und Legitimation des Bundesverfassungsgerichts », in : Jestaedt, M. / Lepsius, O. / Möllers, C. / Schönberger, C. (éd.) : Das entgrenzte Gericht, Berlin, Suhrkamp, p. 281-408.

Möllers, C. / Voßkuhle, A. (2003) : « Die deutsche Staatsrechtswissenschaft im Zusammenhang der internationalisierten Wissenschaften. Beobachtungen, Vermutungen, Thesen ", in : Die Verwaltung, 36 (3), p. 321-332.

Pauly, W. (2008) : « Wissenschaft vom Verfassungsrecht: Deutschland », in : Bogdandy, A. v. / Cruz Villalón, P. / Huber, P. M. (éd.) : Ius Publicum Europaeum, t. II, Heidelberg, C. F. Müller, § 27, p. $463-490$.

Pauly, W. (2011) : «Wissenschaft vom Verwaltungsrecht: Deutschland », in : Bogdandy, A. v. / Cassese, S. / Huber, P. M. (éd.) : Ius Publicum Europaeum, t. IV, Heidelberg, C. F. Müller, § 58, p. 41-80. 
Schlink, B. (1989) : « Die Entthronung der Staatsrechtswissenschaft durch die Verfassungsgerichtsbarkeit », in : Der Staat, 28 (2), p. 161-172.

Schlink, B. (2007) : « Abschied von der Dogmatik. Verfassungsrechtsprechung und Verfassungsrechtswissenschaft im Wandel », in : Juristenzeitung, 62 (4), p. 157-162.

Schmidt-Aßmann, E. (2004) : Das allgemeine Verwaltungsrecht als Ordnungsidee, $2^{\mathrm{e}}$ éd., Berlin, Springer.

Schoch, F. (2007) : « Gemeinsamkeiten und Unterschiede on Verwaltungsrechtslehre und Staatsrechtslehre », in : Schulze-Fielitz (éd.) : « Staatsrechtslehre als Wissenschaft », Die Verwaltung, supplément $n^{\circ}$ 7, p. 177-210.

Schönberger, C. (2006) : «Verwaltungsrecht als konkretisiertes Verfassungsrecht », in : Stolleis, M. (éd.) : Das Bonner Grundgesetz. Altes Recht und neue Verfassung in den ersten Jahrzehnten der Bundesrepublik Deutschland (1949-1969), Berlin, Berliner Wissenschaftsverlag, p. 53-84.

Schönberger, C. (2011a) : «Verwaltungsrechtsvergleichung : Eigenheiten, Methoden und Geschichte ", in : Bogdandy, A. v. / Cassese, S. / Huber, P. M. (éd.) : Ius Publicum Europaeum, t. IV, Heidelberg, C. F. Müller, § 71, p. 493-540.

Schönberger, C. (2011b) : « Anmerkungen zu Karlsruhe », in : Jestaedt, M. / Lepsius, O. / Möllers, C. / Schönberger, C. (éd.) : Das entgrenzte Gericht, Berlin, Suhrkamp, p. 9-76.

Seinecke, R. (2010) : « Richtige Reinheit oder reine Richtigkeit? Rechtslehren nach Hans Kelsen und Karl Larenz », in : Juristenzeitung, 65 (6), p. 279-287.

Starck, C. (2003) : « Societas Iuris Publici Europaei », in : Juristenzeitung, 58 (17), p. 895.

Stürner, R. (2012) : « Das Zivilrecht der Moderne und die Bedeutung der Rechtsdogmatik », in : Juristenzeitung, 67 (1), p. 10-24.

Thym, D. (2011) : « Anmerkung », in : Juristenzeitung, 66 (20), p. 1011-1015.

Trute, H.-H. / Groß, T. / Röhl, H. C. / Möllers, C. (éd.) (2008) : Allgemeines Verwaltungsrecht - zur Tragfähigkeit eines Konzepts, Tübingen, Mohr Siebeck.

Vogel, J. (2012) : «Strafrecht und Strafrechtswissenschaft im internationalen und europäischen Rechtsraum », in : Juristenzeitung, 67 (1), p. 25-31.

Voßkuhle, A. (2006) : « Neue Verwaltungsrechtswissenschaft », in : Hoffmann-Riem, W. / Schmidt-Aßmann, E. / Voßkuhle, A. (éd.) : Grundlagen des Verwaltungsrechts, t. 1, Munich, C. H. Beck, p. 1-61.

Voßkuhle, A. (2010a) : « Das Leitbild des “europäischen Juristen” - Gedanken zur Juristenausbildung und zur Rechtskultur in Deutschland », in : Rechtswissenschaft, 1 (3), p. 326-346.

Voßkuhle, A. (2010b) : « Der europäische Verfassungsgerichtsverbund », in : Neue Zeitschrift für Verwaltungsrecht, 29 (1), p. 1-8.

Wahl, R. (2003) : « Zwei Phasen des Öffentlichen Rechts nach 1949 », in : Wahl, R. : Verfassungsstaat, Europäisierung, Internationalisierung, Francfort-sur-le-Main, Suhrkamp, p. 411-435.

Wahl, R. (2006) : Herausforderungen und Antworten: Das Öffentliche Recht der letzten fünf Jahrzehnte, Berlin, De Gruyter (= Schriftenreihe der Juristischen Gesellschaft zu Berlin, 178).

Werner, F. (1959) : «Verwaltungsrecht als konkretisiertes Verfassungsrecht », in : Deutsches Verwaltungsblatt, 74, p. 527-533. 
Zürn, M. (2001) : « Politik in der postnationalen Konstellation. Über das Elend des methodologischen Nationalismus ", in : Landfried, C. (éd.) : Politik in einer entgrenzten Welt, Cologne, Verlag Wissenschaft und Politik, p. 181-204.

\section{NOTES}

1. Möllers / Voßkuhle (2003), p. 321 sqq.

2. Bogdandy (2011), p. 1 sqq.

3. Voßkuhle (2010a), p. 326 sqq.

4. Pour la doctrine civiliste et la doctrine pénaliste, voir, dans le présent volume, Stürner (2012), p. 10, ainsi que Vogel (2012), p. 25.

5. À cet égard, cf. Bogdandy (2011), p. 4, se référant à Zürn (2001), p. 181 ; à titre complémentaire et pour un exemple tiré du droit administratif comparé, cf. Schönberger (2011a), § 71, n ${ }^{\text {ss }} 50$ sqq.

6. Brohm (2001), p. 1079.

7. Sur ce point et pour la suite de notre propos, cf. Jestaedt (2011b), p. 169 sqq.

8. Ayant déployé une influence considérable : Larenz (1960), p. 189. À ce sujet, cf. Seinecke (2010), p. 283 sq. et en particulier p. 285 sq.

9. La seule Loi fondamentale a donné lieu, à ce jour, à 25 codes commentés, dont deux éditions particulièrement approfondies (le commentaire dit «de Bonn » et le «Maunz / Dürig ») et cinq éditions en plusieurs volumes (le commentaire «Mangoldt / Klein / Starck», le commentaire «von Münch », le «commentaire alternatif», le commentaire "Dreier» et le «commentaire berlinois »).

10. Sur le fait que la science du droit et la pratique du droit emploient un langage commun avec des objectifs différents, cf. Jestaedt (2011b), p. 178 sqq.

11. Pour p. ex. le droit social, cf. Becker (2010), p. 15 sq.

12. À ce sujet, en se référant à la Loi fondamentale, cf. Lerche (2001), p. 340-342 et 342-348.

13. Pour une analyse plus approfondie, cf. Hoyningen-Huene (2008), p. 167 sqq.

14. A ce sujet, cf. Pauly (2008), $\S 27, \mathrm{n}^{\text {os }} 13$ et 18 . Traditionnellement, la doctrine de droit international public n'est considérée comme élément - dépendant - de la doctrine de droit public que dans la mesure où - du point de vue de la discipline scientifique - elle se recoupe avec la science administrative et la science constitutionnelle et/ou - du point de vue des personnes - le scientifique en question possède, outre sa qualité d'enseignant de droit constitutionnel ou de droit administratif, une qualité d'enseignant de droit international public qui le qualifie particulièrement pour se prononcer sur la question concrète à traiter.

15. À la date du 22 novembre 2011, le registre des membres de l'Association comporte les noms de 717 enseignants de droit public venant d'Allemagne, d'Autriche et de la Suisse, ainsi que, pour une poignée d'entre eux, d'autres pays (dont p.ex. la France ou la Grèce). Cf. https:// www.vdstrl.de/mitgliederverzeichnis/ (site consulté le 11 décembre 2019).

16. Pour une analyse approfondie, cf. Schoch (2007), p. 177 sqq.

17. Au sujet de la Cour constitutionnelle fédérale comme "pouvoir fixant des critères de référence ", cf. l'analyse détaillée de Lepsius (2011), p. 159 sqq.

18. De manière fondamentale, cf. Lepsius (2008), p. 103 sqq.

19. À ce sujet, cf. Schönberger (2011b), p. 11 sqq.

20. Pour une analyse plus approfondie, cf. Schlink (1989), p. 161 sqq. ; Schlink (2007), p. 157 sqq. ; Jestaedt (2002), p. 183 sqq.

21. À cet égard, cf. la contribution particulièrement pertinente d'Isensee (1992), § 162.

22. Recueil BVerfGE 61, 82 (108).

23. Hollerbach (1969), p. 51 sqq. 
24. Recueil BVerfGE 7, 198 (205, 207).

25. Au sujet des différentes formes que peut prendre la constitutionnalisation, cf. Jestaedt (2011a), p. 37 sqq.

26. Pour une explication et une critique de la technique de l'interprétation conforme à la Constitution, cf. Lembke (2009).

27. Citation tirée de Löwer (2005), § 70, $\mathrm{n}^{\circ} 126$.

28. Alexy (1985), p. 77 sqq.

29. Formulation employée par Pauly (2008), § 27, $\mathrm{n}^{\circ} 16$.

30. Pour plus de détails, cf. Jestaedt (2010), $§ 1, \mathrm{n}^{\text {os }} 10$ sqq., 13 sqq., 53 sqq. et 68 sqq.

31. Cf. notamment, en ce qui concerne la Seconde Chambre de la Cour constitutionnelle fédérale : Recueil BVerfGE 123, 267 sqq. - Lisbonne, cf. Revue JZ 2009, p. 890 (au sujet de cet arrêt, cf. Gärditz / Hillgruber [2009], p. 872 et Classen [2009], p. 881) ; BVerfGE 126, 286 - Honeywell, cf. Revue JZ 2010, p. 1177 (avec un commentaire de Claus Dieter Classen) ; arrêt du 7 septembre 2011, aff. 2 BvR 987/10, 1485/10, 1099/10 - mesures de sauvetage de l'euro, disponible en ligne sous http://www.bverfg.de/entscheidungen/rs20110907_2bvr098710.html (consulté le 6 décembre 2011 ; 11 déc. 2019), cf. revue JZ 2011, p. 1004 (avec un commentaire de Daniel Thym) ; arrêt du 9 novembre 2011, aff. $2 \mathrm{BvC} 4 / 10,6 / 10,8 / 10$ - seuil des $5 \%$ lors des élections européennes, disponible en ligne sous http://www.bverfg.de/entscheidungen/cs20111109_2bvc000410.html (consulté le 6 décembre 2011 ; 11 déc. 2019) ; en ce qui concerne la Première Chambre, cf. l'arrêt du 19 juillet 2011, aff. 1 BvR 1916/09 - titularité des droits fondamentaux au profit de personnes morales des États membres de l'Union européenne, cf. Revue JZ 2011, p. 1112 sqq. (avec un commentaire de Christian Hillgruber).

32. Terme utilisé par Voßkuhle (2010b), p. 1 sqq.

33. À ce sujet, cf. Schönberger (2011b), p. 59 sqq. ; Möllers (2011), p. 402 sqq.

34. Pars pro toto, cf. Pauly (2008), § $27, \mathrm{n}^{\circ} 17$.

35. Bachof (1963), p. 257, emploie dès 1963 le terme de la « dépendance systématique du droit administratif vis-à-vis du droit constitutionnel ».

36. Wahl (2003), p. 422, décèle dans "l'ouverture envers une orientation vers l'extérieur de l'ordre juridique » l'élément déterminant de la « seconde phase » d'évolution du droit public en Allemagne sous l'empire de la Loi fondamentale.

37. Le président de l'époque de la Cour fédérale administrative marqua fortement ce débat de son empreinte, cf. Werner (1959), p. 527 sqq.

38. Pour une analyse plus approfondie du rôle de la justice administrative (notamment de la Cour fédérale administrative), cf. Schönberger (2006), p. 53 sqq.

39. Terme employé par Wahl (2006), p. 41.

40. Wahl (2006), p. 38.

41. Cf. la contribution paradigmatique de Schmidt-Aßmann (2004), dont le sous-titre est, de manière significative, "fondements et missions de la création de systèmes en droit administratif " (nous soulignons). Dans ce contexte, sur la question de la viabilité d'un concept, cf. Trute et al. (2008).

42. Les volumes publiés sous la direction de Wolfgang Hoffmann-Riem et Eberhard SchmidtAßmann et intitulés «Schriften zur Reform des Verwaltungsrechts " (Documents pour la réforme du droit administratif) : 10 volumes, publiés entre 1993 et 2004, peuvent être considérées comme des contributions qui ont marqué les débats de leur empreinte.

43. Un monument de la littérature relative à la « Nouvelle science du droit administratif » est l'ouvrage publié sous la direction de Hoffman-Riem et al. (2006-2008). Le projet est décrit de manière concise par Voßkuhle (2006), § 1.

44. En ce sens, cf. Pauly (2011), § 58, $\mathrm{n}^{\circ} 20$.

45. Pour une critique du point de vue de la «méthodologie juridique», cf. Krebs (2004), p. 219 sqq. 
46. À titre d'exemple, cf. Pauly (2011), § 58, nº 34 .

47. À ce sujet, cf. Starck (2003), p. 895.

48. À ce jour, quatre volumes de IPE ont été publiés, pour un total d'environ 3000 pages. Le terme de «droit constitutionnel européen commun » a été employé très tôt par Häberle (1992), p. $71 \mathrm{sqq}$.

49. En ce qui concerne le droit administratif comparé, les juristes naviguent alors entre Charybde (le "nationalisme méthodologique ») et Scylla (" l'euphorie de la convergence »); à ce sujet, cf. les travaux fondamentaux de Schönberger (2011a), § 71, $\mathrm{n}^{\text {os }} 50-52$.

50. Cf. l'article 23 de la Constitution dogmatique sur l'Église, Lumen Gentium, qui fait depuis 1983 partie du droit positif ecclésiastique.

51. Il est révélateur à cet égard que le manuel classique relatif à la CEDH commercialisé en Allemagne n'ait pas été rédigé par un Allemand, cf. Grabenwarter (2009).

\section{INDEX}

Schlüsselwörter : Staatsrechtslehre, Dogmatik, Öffentliches Recht, Europäisierung

Mots-clés : doctrine, dogmatique, droit public, européanisation

\section{AUTEURS}

\section{MATTHIAS JESTAEDT}

L'auteur est coéditeur de la Revue JuristenZeitung, responsable pour les questions de droit public et de méthodologie. Il est professeur de droit public et de théorie du droit à l'Université Albert Ludwig à Fribourg-en-Brisgau. Voir aussi la notice suivante. 